\title{
NOTAS SOBRE LOS CONFLICTOS Y CONTACTOS LINGÜISTICOS ENTRE EL MAYA YUCATECO, EL ESPAÑOL Y EL INGLÉS EN LA ZONA DE RÍO HONDO
}

\author{
Jette Bolle
}

Centro de Investigación y Estudios Superiores en Antropología Social, Campeche

\begin{abstract}
AвstrAct: This paper deals with Yucatec Maya, Spanish and English language contact and conflict situations, along the border between Mexico and Belize. First the multilinguistic situation in both countries will be explained. The diglossic situation in which Yucatec Maya, the dominant language, will be explained; the situation in Belize is more marginalized. On both sides of the border Yucatec Maya shows effects of contact with Spanish above all. In the second part of the article examples are provided of some changes within Maya and the influence that Spanish exerts on phonology, morphosintaxis, and lexicon of Yucatec Maya. The examples come from texts compiled by Smailus in Belize and Quintana Roo.
\end{abstract}

Resumen: Este artículo aborda la situación de contacto y conflicto entre el maya yucateco, el español y el inglés en la zona fronteriza de México-Belice. Primero da información de la situación multilingủe de la región. Describe la situación de diglosia en que se encuentra el maya yucateco, la cual es más marginal en Belice. En ambos lados de la frontera se ven los efectos del contacto con el maya yucateco, sobre todo del español. Se dan ejemplos de la integración de elementos españoles en los niveles fonológico, morfosintáctico y léxico en los textos compilados por Smailus en Belice y en Quintana Roo. Además la autora trata de los cambios en el maya regional.

\section{Introducción ${ }^{1}$}

Las lenguas indigenas en México se han descrito sobre todo desde el punto de vista de sus estructuras internas, esto es, en términos de sus sistemas fonológicos y gramaticales. Existen escasos estudios en torno a los contactos lingüísticos con el español y los efectos que este contacto ha producido sobre las propias lenguas (para una crítica, $c f$. Flores Farfán, 1995).
Este artículo describe una parte del proyecto en que se investigan los contactos lingüísticos y sus efectos en las lenguas de la zona del Río Hondo, en la frontera México-Belice. Se trata de un estudio minucioso de los efectos lingüísticos y sociolingüísticos del contacto asimétrico entre lenguas nacionales (español e inglés) y una lengua marginada (maya yucateco), sobre todo en el caso de Belice. La compleja situación lingüistica se presenta como una serie de situaciones

\footnotetext{
${ }^{1}$ Este artículo es una síntesis del proyecto sobre la situación lingüística de la región del Río Hondo, con una revisión más profunda de la bibliografia sobre el contacto lingûistico del maya yucateco y con las notas de los primeros recorridos de campo en enero y septiembre de 1996.
} 
diglósicas. Esto es porque cada una de las lenguas se asocia con distintas funciones y valores. Esto tiene su expresión, por ejemplo, en distintos tipos y grados de bilingüismo y en los procesos de cambio asociados, lo que básicamente tiene que ver con el grado de influencia e interpenetración de las lenguas en contacto. Para ello se busca ejemplificar la situación del maya yucateco en México y en Belice, tras investigar distintas comunidades. Se considera que de esta manera será posible analizar el grado de influencia de las lenguas en contacto en situaciones sociolingüísticas complejas.

La situación del maya yucateco es la de una lengua marginada, con mucha presión de la(s) lengua(s) dominante(s). He sugerido que en Belice la lengua maya se encuentra en una situación relativamente más marginal que en México, lo cual se expresa por un número relativamente menor de hablantes (véase cuadro 1).

En ambos lados de la frontera se habla el maya en menor porcentaje que en la "zona maya" de la península. En México eso se explica en parte como consecuencia de la migración hacia Río Hondo desde distintas partes de la República por personas que no son hablantes de maya. En Belice se ve que el porcentaje es consecuencia del cambio de la lengua primaria; los mayas yucatecos dejaron de usar el maya en favor del español (y a veces incluso del inglés). El rápido desplazamiento se ve en el porcentaje más alto de $21.8 \%$ en 1946 (Brockmann, 1977b).

Lingüisticamente hablando, casi no se conoce nada sobre el maya yucateco hablado en la zona, aunque el papel de este grupo en el desarrollo de la región es reconocido (cf., por ejemplo, Macías, 1985; Bolland, 1992). Generalmente se afirma que el maya yucateco se caracteriza por tener poca variación dialectal (cf., por ejemplo, Pfeiler, 1995). El único trabajo sobre el maya yucateco de Belice que he encontrado es una compilación de cuentos de Smailus (1975). El autor hace
Estudios de Cultura Maya. Vol. XXI, 2000

Instituto de Investigaciones Filológicas/

Centro de Estudios Mayas, UNAM

ISSN: 0185-2574 solamente unos comentarios generales sobre la situación del maya, sin llegar a la investigación estrictamente lingüistica. En la segunda parte de este artículo se darán los resultados del análisis del contacto maya-español en los textos de Smailus.

Existen algunos estudios sociolingüísticos sobre las regiones en que se habla el maya yucateco, en los que se describen las comunidades bilingües mayas en términos de uso y funciones de las lenguas, en relación con los factores sociales en las mismas comunidades. Jiménez Peraza (1982) llevó a cabo un estudio en Yucatán sobre la lealtad hacia la lengua maya y Pfeiler (1988) hizo una comparación sobre el uso del español y del maya en dos comunidades. Kümmer (s.f.) describe las actitudes hacia las dos lenguas en Yucatán. Los estudios en Belice se centran sobre todo en la cuestión de la identidad de los hablantes. Por ejemplo, sobre el distrito Cayo, en Belice, Le Page y Tabourett-Keller (1985) hacen hincapié en el criollo y el español, y existen estudios sobre el distrito Orange Walk (Brockmann, 1977 a y b) y sobre el distrito Corozal (Koenig, 1975, 1980). En dichos trabajos se emplean sobre todo el cuestionario y las entrevistas, y en el caso de Koenig y Pfeiler se combinan con observaciones personales. Hay también trabajos sobre los efectos lingüísticos del contacto entre el maya yucateco y el español en los cuales se menciona el estudio de documentos desde la época colonial de Karttunen (1985) y los trabajos de Pfeiler (1995, 1996), donde describe la variación lingüística de la península y aborda el tema del cambio en el maya.

\section{Descripción general del uso de las lenguas}

\section{En México}

Sobre los pueblos del lado mexicano del Río Hondo no he encontrado ningún estudio o 


\section{Cuadro 1}

Porcentaje y número de hablantes del maya yucateco

\begin{tabular}{|c|c|c|}
\hline Región & Hablantes de maya yucateco & Fuentes \\
\hline México & 713520 & XI Censo 1990 \\
\hline Quintana Roo (+490 000) & $26.8 \%(120846)$ & XI Censo 1990 \\
\hline Río Hondo & $5.6 \%$ (OPB 20000 -ini) & CIQRO, 1993 \\
\hline Botes (235) & $50 \%(117)$ & \\
\hline Cacao (1382) & $50 \%(669)$ & \\
\hline Coyocol (1890) & (muchos) & \\
\hline Calderón (211) & $15 \%(30)$ & \\
\hline Belice $(+200000)$ & $6.8 \%(+13000)$ & Censo 1980 \\
\hline Patchakan (950) & $10 \%(95)$ & CIQRO, 1993 \\
\hline Yo Chen (400) & (ancianos) & \\
\hline San Pedro (300) & $70 \%(210)$ & \\
\hline San Narciso (300) & (adultos) & \\
\hline San Román (370) & 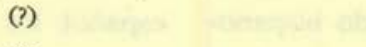 & 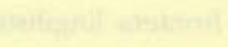 \\
\hline San Antonio (327) & (?) & \\
\hline Santa Cruz (160) & $95 \%(152)$ & 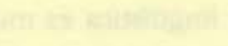 \\
\hline Trinidad (510) & $15 \%(76)$ & 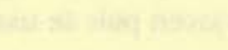 \\
\hline August Pine Ridge (1197) & $10 \%(120)$ & 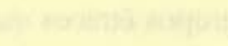 \\
\hline San Felipe (835) & $30 \%(252)$ & 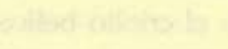 \\
\hline San Victor (529) & (ancianos) & \\
\hline Orange Walk (+ 11350$)$ & $1.7 \%(+190)$ & Brockmann, 1977 \\
\hline Corozal (+ 15 504) & $3.8 \%(+600)$ & Brockmann, 1977 \\
\hline
\end{tabular}

- Incluyendo hablantes de kekchí, maya mopán y maya yucateco.

compilación lingüística. La mayor parte del material sobre el maya yucateco se recopiló en Yucatán o en Quintana Roo. En el siglo xix un grupo de mayas de Chichanhá ${ }^{2}$ y muchos refugiados de la Guerra de Castas se establecieron en la zona de Río Hondo y en Belice (véase Bolland, 1992, y Macías, 1985). Estas comunidades se encuentran en un proceso de desplazamiento lingüístico más avanzado, en unos pueblos más intenso que en otros. En México se trata sobre todo de la relación entre el español y el maya, y cada vez más del inglés como segunda o tercera lengua, sobre todo en la medida en que uno se acerca a Belice. En la zona se entiende el inglés, entre otras razones por las históricas y de comercio. Mucha gente expresa su interés en aprender inglés como forma de avanzar económicamente al encontrar trabajo en el turismo. Los cálculos oficiales acerca del número de hablantes mayas varían, pero en la

\footnotetext{
${ }^{2}$ Los mayas de Chichanhá se opusieron a los mayas de la Santa Cruz. Después de ataques de parte de éstos, los mayas de Chichanhá tuvieron que acercarse más hacia el territorio inglés y establecieron el pueblo Icaiché. Este siglo, el pueblo tenía que trasladarse otra vez, debido a la reforma agraria, y actualmente gran parte de los habitantes reside en Botes.
} 
zona fronteriza del lado mexicano la lengua indígena se habla todavía en algunas comunidades, las cuales están representadas en el cuadro 1. Se habla de porcentajes de hablantes sin especificar sexo, edades o pericia (CIQRo, 1993). En Botes se afirma que la lengua todavía es hablada por la mayor parte de la población. Pero yo misma he notado que las generaciones nuevas dejan de entender y hablar el maya yucateco, lo que supongo una tendencia general en la zona (Bolle, diario de campo, 1996).

\section{En Belice}

Éste es el único país de Centroamérica en el que el inglés es la lengua oficial. Por ello constituye una frontera lingüística con el mundo hispanohablante, lo cual tiene efectos únicos. La situación lingüística es muy compleja, puesto que en este joven país se usan las lenguas de los distintos grupos étnicos que se establecieron en la región: el criollo beliceño, el español, el garífuna, el maya yucateco, el maya mopán, el kekchí, el inglés, el alemán, el hindú, el cantonés y el árabe, entre otras. Debido a la convivencia de estos grupos étnicos en un espacio relativamente pequeño y por las mezclas entre los grupos, Belice constituye un verdadero caleidoscopio de lenguas y culturas. Según el censo de 1980, la lengua con el mayor número de hablantes en Belice es el criollo beliceño. Sin embargo, recientemente, debido a la inmigración hispanohablante en el norte, el español es hablado por la mayor parte de la población. En las fronteras se tiende a utilizar más el español en la vida cotidiana, aunque se requiere el inglés o sus variantes locales para la comunicación interétnica y la interacción con las instituciones estatales. Más aún, esta naturaleza oficial del inglés implica que se utilice en la educación, en situaciones públicas, además de ser la lengua de comunicación turística - el turismo es una de las princi-
Estudios de Cultura Maya. Vol. XXI, 2000

Instituto de Investigaciones Filológicas/

Centro de Estudios Mayas, UNAM

ISSN: 0185-2574 pales fuentes de ingresos del país-. Dadas estas condiciones, si bien el inglés estándar no es la lengua materna de ninguno de los grupos étnicos de Belice, su importancia es creciente. Con esto se sugiere que la mayor parte de la población beliceña es por lo menos bilingüe, si no es que trilingüe, situación cada día más común en general.

En los pueblos mayas del Río Hondo el español suele ser hablado por toda la población. Esto se debe a las relaciones históricas de los mayas yucatecos de Belice con México y a la cercanía geográfica de países hispanohablantes. En los pueblos, el maya tiende a distribuirse generacionalmente, pues los ancianos todavía hablan y usan el maya y entienden un poco del español; los padres entienden (un poco) el maya y usan el español como lengua primaria, y los niños tienen el español como lengua materna. Eso sugiere que el español es prácticamente la lengua primaria de los pueblos.

\section{Pluriglosia}

Una situación en la que en una comunidad se jerarquizan las lenguas (o variantes de una misma lengua) por funciones y dominios "altos" y "bajos" en los que se manifiestan relaciones de dominación y poder, se puede considerar diglósica. En Belice el español y el inglés tienen en general una posición hegemónica en comparación con el maya. Una manifestación palpable de estas situaciones diglósicas se produce en la zona fronteriza, donde las lenguas hegemónicas son las lenguas de contacto y comercio entre los dos países.

En situaciones de diglosia la influencia de una lengua sobre otra en muchos casos implica una influencia unidireccional sobre la lengua marginada, expresión de las fuerzas sociales que se materializan en un mayor status de las 


\section{Figura 1. Las posiciones de las lenguas en la región de Río Hondo}

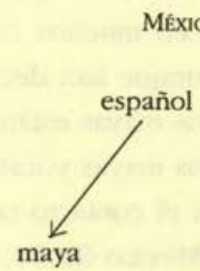

lenguas hegemónicas. Una representación simplificada de las posiciones de las lenguas se provee en la figura 1. Para los mayas que huyeron de México hacia Belice, se observa que el español compite ahora con el inglés, siendo la lengua oficial y la lengua de la educación, además de ser lengua hegemónica, con implicaciones en las relaciones económicas y sociales. En las comunidades mayas se ha aprendido espanol por el mayor status de esta lengua, pero cada vez hay más necesidad de aprender el inglés, sobre todo en el contexto económico. En Belice el maya es considerado "inferior", a diferencia de México. Según Brockmann (1977b), el maya yucateco tiene un status bastante bajo, incluso para muchos hablantes dentro de las comunidades.

Brockmann habla de diglosias entre el español y el inglés. Los hispanohablantes tienden a usar el inglés en situaciones oficiales, por ejemplo en los contactos con los autoridades y para la educación. La relación entre el español y el inglés es más compleja, en vista de que ambos tienen status en el norte de Belice. En el estricto sentido del término diglosia, el inglés, por ser usado en la escritura, la educación y en situaciones oficiales, es la lengua "alta". Pero en el norte el español es la lengua hablada por la mayoría y también tiene cierto status, debido, entre otras cosas, a los vínculos con los países hispanohablantes.
BELICE

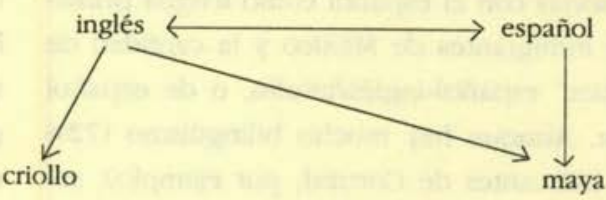

Koenig (1975) hace la distinción entre el inglés estándar —que como lengua oficial tiene poder y status - y las otras lenguas como lenguas de solidaridad. Sin embargo, no describe las diferencias entre lenguas con menos status, esto es, como el status más bajo y las actitudes negativas acerca del maya yucateco y el criollo; tampoco trata el status relativamente más alto del español. ${ }^{3}$

En Belice existe un continuo entre el inglés y el criollo beliceño. Sin embargo, en los pueblos mayas no se habla el criollo (Brockmann, 1977b, y mi propio trabajo de campo). Por ello, el criollo no tiene mucha influencia en la situación lingüística del maya yucateco.

El maya yucateco, entonces, está en una relación con dos lenguas hegemónicas. Sin embargo, en el nivel social la relación entre el español y el maya puede llegar a invertirse, tal como el uso del maya en situaciones públicas en que los hablantes rechazan el status inferior de la lengua e incluso buscan promoverla (Brockmann, 1977a).

Por las múltiples relaciones entre las lenguas, sería más adecuado hablar de una situación pluriglósica en la que cada lengua tiene sus funciones y dominios con respecto a la otra. Por la complejidad de la situación sociolingüística en un espacio relativamente pequeño, prefiero usar el concepto de caleidoscopio, que considera la existencia de multiples variantes y variables de uso en situaciones fragmentadas.

\footnotetext{
${ }^{3}$ Hasta la llegada de gran número de criollos, el español era la lengua dominante en el norte y fue usado incluso por los criollos, a quienes llamaron, por la adaptación lingüística y cultural, "Black Spanish" (Koenig, 1980). Estudios de Cultura Maya. Vol. XXI, 2000 
La conclusión básica del trabajo de Koenig (1975) es que la lengua que más se habla en la zona es el español, en virtud del gran número de personas con el español como lengua primaria, los inmigrantes de México y la cantidad de "bilingües" español-inglés/criollo, o de español y maya. Aunque hay mucho bilingüismo $(72 \%$ de los habitantes de Corozal, por ejemplo), según la autora no hay mucha comunicación entre los distintos grupos étnicos, por las actitudes negativas, entre otras de parte de los mayas o de los hispanohablantes hacia los criollos y de parte de los no mayahablantes hacia los mayahablantes.

En las situaciones de comunicación interétnica, la lengua usada se define en cada uno de los contactos personales. Se pueden encontrar procesos de "negociación" donde los papeles de los hablantes y su status influyen en las lengua(s) que se usa(n) en la conversación. Sin embargo, existen algunas situaciones en que una lengua específica se usa casi siempre, como por ejemplo en situaciones oficiales (gobierno) y en la escuela secundaria, donde se usa inglés "estándar". Se observa que en muchos casos los maestros mismos no manejan el inglés con mucha habilidad.

En cuanto a los hablantes de maya, Koenig llega a la conclusión de que la gente de los pueblos deja de hablar el maya en favor del español, y así se adaptan a una etnicidad de hispanohablantes (mestiza). Esta conclusión es demasiado fuerte, porque no se puede relacionar unidimensionalmente la etnicidad con la lengua materna, y de hecho la noción de etnicidad es más compleja y se define con base en distintos factores. Le Page y Tabourette-Keller (1985) hicieron un estudio sobre la identidad en el distrito Cayo de Belice basado en textos y entrevistas. Ellos critican la relación lengua $=e t n i a=$ identidad, porque los jóvenes se perciben a sí mismes como mixed (mezclados) $x_{1}$, desde luego

Instituto de Investigaciones Filológicas/

Centro de Estudios Mayas, UNAM

ISSN: 0185-2574 separan la lengua materna y la etnicidad. La crítica se refuerza por el hecho de que las personas se autodenominan en muchos casos como mayas y maasewales, aunque han dejado de hablar el maya. A veces los mayas están conscientes de la relación con los mayas yucatecos de la península por mantener el contacto con sus parientes que residen en México (Bolle, trabajo de campo, 1996).

No se puede negar que el maya esté en un proceso de desplazamiento en que los hablantes en general adoptaron el español como lengua materna, en vez del inglés, que es la lengua oficial. Comparada con el español, el inglés y el criollo, la lengua indígena está en la situación más vulnerable; por ello existen más efectos del contacto y también proclividad al desplazamiento.

\section{Desplazamiento y actitudes de los hablantes}

El desplazamiento del maya yucateco ocurre rápidamente por generaciones en comunidades en ambos lados de la frontera. El desprestigio hacia el maya se ve en que los hablantes a veces no admiten hablar el maya, y solamente con vergüenza indican a los hablantes. Las personas que tienen más interés en "el rescate" del maya sostienen, al contrario, que casi toda la gente en una comunidad habla y usa el maya, aunque sus propios hijos y nietos ya no lo hablan. Se ve que existen paradojas y ambivalencia en los comentarios de los hablantes, lo que puede indicar la existencia de una identidad fragmentada.

$\mathrm{Si}$ bien en Belice encontramos situaciones de lealtad hacia el idioma maya, se considera que el maya "legítimo" proviene de México, lo cual es congruente con la historia social de estas comunidades: "En Yucatán hablan maya muy diferente (mejor), aquí el maya está mixoteado" (mujer maya, 65 años, Chan Pine Ridge, Belice; 
cit. en Bolle, diario de campo, 1996). Este testimonio alude a la influencia que el inglés ejerce sobre el maya y el español -manifiesto en el propio fragmento en el anglicismo mixoteado (del ingés to mix 'mezclar') - y muestra la conciencia de los hablantes mayas sobre la situación lingüistica. En el mismo pueblo un hablante de más de 80 años comentó que el maya que habla la gente tiene muchas palabras provenientes del "castilla" y desde luego inglesas.

Las apreciaciones sobre la cuestión de la habilidad en maya, es en general la siguiente. En muchos casos, la gente dice que sus padres hablan o hablaron maya entre ellos, pero prefieren hablar español con sus hijos. Algunas personas en los pueblos "admiten" entenderlo, pero dicen que cuando tratan de hablar maya se les "atraviesa la lengua (española o inglesa)" (Bolle, trabajo de campo, 1996).

Según Pfeiler (1995), los hablantes de maya en Yucatán distinguen entre dos variedades de maya. La primera es llamada la bach maya (la muy maya) y es descrita como la lengua verdadera, antigua y pura. Según los hablantes del maya, esta variedad es hablada por los antiguos y los mayeros, en especial de la zona de Valladolid. La variedad llamada el xe'ek' (ensalada) es hablada por los jóvenes de origen rural que trabajan en Mérida. Se califica como mestiza, mezclada con el español. Aquí cabe mencionar que en la situación actual del maya yucateco es dificil encontrar el uso del maya sin que se presenten por lo menos algunos efectos del español, aunque sea nada más en el léxico. Por ello supongo que la mencionada diferencia entre las variantes de maya se basa en una diferencia gradual del uso de elementos españoles y del uso más variable de más estructuras del maya.
De hecho los hablantes de los pueblos mayas del Río Hondo también se expresan en distintas variantes del maya. Un hablante me explicó que en los pueblos existe el maya "puro" y el maya "mestizo". Según este hablante, se notan las diferencias sobre todo en el vocabulario. Los llaman el maya "verdadero" y el maya "mestizo", tal como plantea Pfeiler. ${ }^{4}$ La conciencia de estas variedades y la base lingüistica plasmada en distintos estilos, registros o sociolectos, debería ser investigada con mucho más profundidad en las distintas comunidades. Por lo tanto, por la situación más marginada en que se encuentra el maya de Belice, la influencia de las lenguas hegemónicas es muy fuerte. Entre otras cuestiones, se puede ver que el maya presenta influencia del español (y a veces del inglés) en el léxico, la morfología e incluso en la gramática. Existe una influencia del maya yucateco hacia el español o el inglés, que, por el poco espacio, no se abordará en este artículo.

\section{Los efectos lingüísticos}

Como he mencionado, existen pocos estudios sobre el contacto maya-español y éstos describen otras regiones. Además, en muchos casos se trata del español hablado en Yucatán. Smailus (1975) compiló narraciones de tradición oral en Belice en San Víctor, distrito Orange Walk (SV), en San Antonio, distrito Cayo (SA), y en Felipe Carrillo Puerto, Quintana Roo (FCP). Según él, existe más influencia del español en Belice, sobre todo en la zona del Río Hondo. Smailus menciona la pérdida de funciones gramaticales, como el uso de los clasificadores, o la variabilidad de estas funciones, pero no profundiza al respecto. Según él,

\footnotetext{
4 Los hablantes dan los mismos ejemplos para mostrar las diferencias en el vocabulario, como en el trabajo de Pfeiler. Por ejemplo: "los mestizos usan k'aanche' mientras que los que hablan el maya verdadero dicen kisi'che' para banquillo" (Don N., Botes; Bolle, trabajo de campo, 1996).
} 
los efectos del español no se ven tanto en la presencia de elementos españoles, sino en el uso de un maya menos variable y con menos empleo de las construcciones propias del maya, lo que antes he vinculado con el maya "puro". Aquí me voy a concentrar en los elementos españoles que entraron al maya, para tratar de esbozar las estrategias y características del contacto con el español.

En San Víctor, Smailus solamente recopiló cuentos de hablantes que tenían poco o nulo conocimiento del inglés, por lo que este aspecto no está ni siquiera perfilado. ${ }^{5}$ El material consiste en siete cuentos de tres hablantes de entre 14 y 26 años, con un total de casi 13000 palabras. En San Antonio los hablantes más jóvenes saben un poco de inglés. En este pueblo se recopilaron ocho cuentos de cinco hablantes con unas 5000 palabras. Los cuentos de San Víctor, principalmente, tienen motivos españoles. Se trata de cuentos sobre reyes, princesas, caballos, etc. Por ello, la base de los cuentos es europea, y esto influye en la cantidad de préstamos. Aquí afirmo que la integración también se ve en textos de tradición oral con temas mayas y que la diferencia es sobre todo de grado. ${ }^{6}$ En San Antonio algunos cuentos tratan de la fundación del pueblo. En Felipe Carrillo Puerto se habla de la Guerra de Castas, y además se recopilaron cuentos con temas tradicionales o europeos (cinco cuentos con aproximadamente 10000 palabras); es notable que aunque en este poblado también se ven las interferencias (verbos, sustantivos, adjetivos, etc.), sobre todo los hablantes con poco conocimiento del español toman palabras menos distintas en préstamo, o sea que el repertorio es más restringido. Sin embargo, lo que no se profundiza en este artículo son los efectos en el maya mismo, lo cual se analiza más a fondo en Bolle (s.f.).

\section{Integración de elementos españoles}

En los cuentos de Smailus (1975) se pueden distinguir ciertos rasgos en el maya debidos al contacto con el español: ${ }^{7}$ el uso abundante de elementos españoles, como sustantivos, adjetivos, verbos, conjunciones y marcadores discursivos, que juntos constituyen $10 \%$ del corpus. En los siguientes ejemplos se pueden ver las maneras en que el maya incorpora elementos del español:

1a) Tu má.n u ká.t ma' minaan untú. má.c ecstrrà.nyo kuchahaan. ${ }^{8}$

SV A3s pasear 3 s preguntar $\mathrm{N}$ no-hay un-C hombre extraño llegado.

"Están andando preguntando si ha llegado un hombre extraño".

b) Ca dzaab umppé. simin yét umppé nuxi' espà.da yéteh u dzò.n.

SV Y dieron un-CI caballo con un-CI vieja espada con PS carabina.

\footnotetext{
${ }^{5}$ En los textos nada más aparecen algunas palabras del inglés: creek (arroyo) y lonch (almuerzo), bedlait (lámpara en la cabeza), etc. Las palabras pueden haber llegado en el léxico maya por medio del español.

${ }^{6}$ Esto se sostiene por una revisión superficial de otras compilaciones. Aunque gran cantidad de los textos se ha publicado en un maya "purificado", con omisión de las palabras españolas.

${ }^{7}$ En el análisis no se trata de distinguir entre el proceso de cambio de código y los préstamos. Teóricamente se pueden distinguir estos procesos, pero en el nivel de los textos es muy dificil separarlos. Una indicación del status de préstamo puede ser la integración de palabras en la morfosintaxis de otro idioma. Para un análisis de la separación de los procesos, cf. Bolle (1994).

${ }^{8}$ Esta escritura está basada sobre todo en la escritura colonial, aunque ampliada con ciertas distinciones fonológicas (véase Smailus, 1975). Las traducciones están basadas en la traducción dada por Smailus:

v: vocal larga, vv: vocal rearticulada, cc: consonante glotal, c: k, k: k', ch: ̌̌ , ch': .̌́, tz: ts, dz: ts', x: s, n: n, rr: r.

A: aspecto, I: intransitivo, T: transitivo, PS: posesivo, P: preposición, CI: clasificador inanimado, CA: clasificador

animado, D: demostrativo, ADJ: marcador plural adjetivo, R: reflexivo, pl: plural, s: singular.
Estudios de Cultura Maya. Vol. XXI, 2000 
"Y le dieron un caballo con una espada grande y una carabina".

c) mehen ch'ilih che'o' rregulartacoo'.

SV pequeño ramas árbol-D rregular-ADJ-pl "pequeñas ramitas regulares".

El ejemplo 1a manifiesta un adjetivo español en el orden artículo-sustantivo-adjetivo, mientras que el orden canónico del maya es adjetivo-sustantivo. En contraste, los adjetivos mayas que aparecen con sustantivos españoles se anteponen, como puede verse en ejemplo $1 b$, lo que sugiere que el orden cambia solamente por la presencia de adjetivos españoles. En los textos de San Víctor se ve la variación de adjetivos mayas antepuestos y adjetivos españoles casi siempre pospuestos, aunque la cantidad de adjetivos españoles es relativamente baja comparada con ejemplos de sustantivos. En San Antonio y Carrillo Puerto no aparecen muchos adjetivos mayas con sustantivos españoles. Solamente una vez en los textos aparece el marcador de plural para adjetivos -tak, como en el ejemplo 1c. Esto se basa, entre otras cosas, en la baja frecuencia de -tak en general en los adjetivos mayas en los textos.

En el ejemplo 2 se puede ver que los adjetivos españoles se utilizan en predicaciones, igual que los adjetivos mayas:

2) Wi'ihoo'.'Ukahoo'. Sú.syoo'.

$\mathrm{SA}$ (tener) hambre-3pl. (tener) sed-3pl. sucio-3pl

"Tenían hambre. Tenían sed. Eran sucios."

El hecho de que aparezcan relativamente pocos adjetivos puede ser explicado en parte por las dificultades con la posición del adjetivo o por las distintas reglas de su uso, como por las diferencias en el uso de predicaciones.

Un tipo de elementos españoles que apare- cen mucho más frecuentemente son los sustantivos. Muchas veces los sustantivos españoles llevan los marcadores del maya, como los elementos demostrativos en $3 \mathrm{~b}$ :

3a) e iglè.syaooba'.

FCP D iglesia-pl-D

"estas iglesias".

b) tu wè.bos.

SA "a sus huevos".

c) yétel miil 'ò.mbres cu maachloo'.

y mil hombres ASP-3 agarrar-3pl

FCP "y mil hombres fueron agarrados".

El plural de los sustantivos españoles se manifiesta de distintas maneras. La palabra española puede aparecer solamente con el plural -o'ob del maya, como en 3a, o aparece con doble plural, como en soldà.dosoobo' (San Víctor). A veces, en español el plural es con -(e)s, como en el ejemplo 3b. Esto ocurre más frecuentemente en combinación con numerales españoles, como en el ejemplo 3c. En los textos existe aparentemente una variación en el uso de plural. Parte de la explicación puede ser que en el maya no es obligatorio marcar el plural. Debido a que el plural español nunca aparece con sustantivos mayas, no se puede hablar de un cambio en el sistema maya.

Otras estructuras en las que se pueden encontrar sustantivos son las frases nominales, como en los ejemplos $4 \mathrm{a}$ y $4 \mathrm{~b}$, y en una predicación, como en el último ejemplo:

\section{4a) las 'ònse del día.}

SV "Las once del día".

b) mí las 'ònse kì.ne'.

SV más-o-menos las once día. "Más o menos las once del día".

c) no maalo' cómpanyè.robeche'".

SA $\mathrm{N}$ bueno compañero-2s-F "no eres buen compañero". 
Se ve que las frases nominales temporales se expresan muchas veces en español, lo que refleja la pérdida del sistema numérico del maya. En los textos se usan nada más (b)un- (uno), ka- (dos) oox-(tres) y kan (cuatro), junto con los clasificadores numerales, mientras que aparecen los numerales españoles, como seis, siete, cien, mil, millones y docena. A veces, como en el ejemplo $4 \mathrm{~b}$, se encuentra mezclada la expresión temporal del español con el maya. En ésta no aparece la preposición española, lo que sugiere que la estructura básica es la del maya; también se puede decir esto de los ejemplos de predicación.

El género solamente se da en el maya por medio de los marcadores ( $a$ ) $b$ - (masculino) e (i) $x$ - (femenino), en sustantivos y adjetivos animados. En los ejemplos $5 \mathrm{a}$ y $5 \mathrm{~b}$ se ve que estos marcadores se usan con palabras españolas que ya expresan el género femenino por medio de la $-a$ final, como en el ejemplo 5b:

5a) xnù.c rrey.

FCP F-vieja rey.

"reina".

5b) untú. chan xbyebi.tae'.

SV un-CA pequeña F-viejita. "una viejita".

Esto sugiere que en el maya las palabras españolas pierden la connotación de género, quizá porque en el maya se usan los sufijos $-o^{\prime} /-a^{\prime}$ de los demostrativos, y posiblemente por falta de conocimiento de la palabra española femenina en Carrillo Puerto, porque $(x)$ reina sí aparece en los cuentos de Belice.

Un cambio en el maya yucateco se puede observar en el uso de los clasificadores. Smailus mismo menciona que hay cambios, pero no profundiza al respecto. Tradicionalmente el maya tie- ne muchos clasificadores, pero actualmente se usa sobre todo -túul con animados y -p'éel con inanimados (cf., entre otros, Barrera Vásquez, 1977; Briceño Chel, 1993). En los textos de Belice se encuentran varios ejemplos del uso de -p'ée( $l$ ) con seres animados, como en el ejemplo 6:

6a) umppé. simin.

SV un-CI caballo. "un caballo".

b) Volarmahe' umppé. chan ch'ích', umppé. cham pá.lomah.

SV volar-I un-CI pequeña abuela, un-CI pequeña paloma.

"Voló un pajarito, una palomita".

De hecho, el cambio de clasificadores se encuentra en mucho menos proporción en San Antonio. No he encontrado evidencia del cambio en textos de Carrillo Puerto, lo que sugiere que el cambio es reforzado por el contacto con el español. El uso de p'é(el) con animados indica que el sistema de clasificadores sigue disminuyendo hacia un sistema solamente con un elemento para todos los sustantivos. El proceso es reforzado por el contacto con el español, lo cual se ve por la frecuencia más alta en San Víctor, aunque este aspecto debería ser estudiado más a fondo.

Un elemento del español que entró al maya es el diminutivo -ito (Pfeiler, 1995). Mientras que la integración morfológica es muy frecuente, en el corpus de Smailus el elemento -ito ocurre con sustantivos españoles y sólo algunas veces con sustantivos mayas, como en el ejemplo 7 :

7) n.ohch u ppó.c i lèti'e' chinaní.to!

SV grande PS sombrero y él pequeño-ito "(Qué) grande su sombrero y él está tan chiquito!"

\footnotetext{
- Nota del editor: Aquí la palabra significa 'pájaro', lo que hace más coherente la frase. Estudios de Cultura Maya. Vol. XXI, 2000 
Una categoría que aparece con más frecuencia en los textos son los verbos españoles, como en el ejemplo 8:

\section{8a) Pues teelo' ca 'énamoràarmah e chan xiipalo'.}

SV pues allá y enamorar-M D pequeño muchacho.

"Pues allá se enamoró el pequeño muchacho"

8b)* u dé.fendèrn ubah/tiaal a depen derrticaba

SV/FCP $3 s$ defender-T $3 s-R /$ para $2 s$ defender-T $2 s-R$

"él se defienda/ para defenderte"

8c) Yan c 'énkomtrarntik, baax 'ù.ch ti' ac màmao'

SV A $1 \mathrm{pl}$ encontrar-T, qué pasar-0 P PS mamá-D

"Vamos a encontrar, qué pasó a nuestra mamá."

Los ejemplos muestran que los verbos españoles normalmente se usan en infinitivo, al que se añaden elementos morfológicos del maya, como por ejemplo -nah. En 6 a el verbo pierde la marca de reflexividad, mientras que en el ejemplo $6 \mathrm{~b}$ se usa el pronombre reflexivo $-b a(b)$. Se nota que los hablantes buscan distintas maneras de reconciliar las diferencias entre las dos lenguas respecto del uso de reflexivos.

En el maya yucateco, la distinción entre verbos transitivos e intransitivos se marca obligatoriamente. En el uso de verbos españoles se hace esta distinción. En 8 a se marca el verbo intransitivo con -nah. El elemento que se usa con los verbos españoles transitivos es -tik (también en las formas $-t,-k$ ), como en el ejempo 8c. Los verbos españoles se usan en los textos del siglo xvin con estos marcadores (Karttunen, 1985). El primer verbo de este tipo en los textos coloniales es firmartik (firmar), y después se encuentran ejemplos con -na, por ejemplo confersarna (confesar) (Karttunen, 1985). A mediados del siglo xix el uso de verbos españoles con los marcadores $-t(i k)$ o $-n a$ era ya común. En los textos de Smailus la mayoría de los verbos lleva uno de estos marcadores. Sin embargo, también se encuentran verbos españoles con otros tipos de marcadores (por ejemplo $-m$ ), e incluso sin marcadores del maya, como en el ejemplo 9:

\section{9a) Bweno ca dzò.c u gá.narr tum bey. \\ FCP bueno y ASP 3 s ganar entonces así "Bueno, y acaba de ganar así." \\ 9b) mim bin ségerr inw ò.cl \\ SV N-1s FUT seguir/volver 1s entrar "No voy a volver a entrar".}

La ausencia de marcadores indica intransitividad de la acción en muchos casos, pero muestra rasgos aspectuales en el uso de seguir en el sentido de 'continuar la acción' o 'volver a ella'. Sin embargo, este aspecto debe estudiarse con más cuidado.

Un fenómeno muy destacado es el uso de marcadores discursivos españoles, incluso en los textos de los hablantes que casi no hablan español. Son el tipo de elementos más frecuente en los textos, por ejemplo pwes (pues), pero(b) (pero) y bweno(b) (bueno). Brody (1987) describe cómo el uso de conjunciones, marcadores discursivos y expresiones dubitativas españolas se encuentra en muchas lenguas mayas y le llama un proceso "areal", que es típico de ellas. Sin embargo, este fenómeno se ha descrito en muy diversos estudios de lenguas en contacto, y comparado con otros corpus, se usan

- Nota del editor: Si este texto maya está correcto, la traducción debe ser depender y no defender en el texto b. 
mucho más marcadores discursivos de otra lengua en el maya (entre otros, véase Bolle, 1994, sobre el sranan y el holandés; Myers-Scotton, 1993, sobre el suahili y el inglés). La facilidad con la que se toman en préstamo estas partículas se explica, entre otras causas, por la relativa independencia y transparencia gramatical de estos elementos, que siempre aparecen en posiciones principales de la frase sin elementos morfosintácticos. Otros elementos que se usan son las preposiciones y las conjunciones. Karttunen (1985) da unos ejemplos de las partículas tomadas en préstamo del español, como por ejemplo de, en, y/e y a. Según ella, al principio estos rasgos no fueron analizados como elementos, sino tomados en préstamo junto con frases nominales. En el siglo xIx aparecen partículas como hasta, entonces. En la época moderna se usan muchas más particulas distintas. El uso de preposiciones españolas no es frecuente en los textos actuales y ocurre en algunos casos en frases hechas como en 10b, en las frases expresadas por un hablante monolingüe:

10a), pero cy aalaa ti' antes bin waye'.

SV pero A-3 dicen a- 3 s antes ir aquí. "pero le dicen antes de ir de aquí".

10b) Pwes chen de rrepè.nteh umppé. kì.ne',

FCP pues sólo de repente un-CI día, "Pues sólo de repente (en) un día",

\section{Adaptación fonológica}

de los elementos españoles

Respecto de la adaptación fonológica de las palabras españolas, se pueden describir muchos procesos, pero en general las palabras no se adaptan tanto al maya como ha descrito Karttunen (1985) para la época colonial. La autora describe, por ejemplo, la sustitución o confusión de consonantes españolas $(d, r$ y $\bar{r}, g, f)$, epéntesis, metáEstudios de Cultura Maya. Vol. XXI, 2000

tesis y la simplificación de agrupaciones de consonantes. Aquí solamente quiero mencionar algunos fenómenos encontrados en el trabajo de Smailus, dejando un análisis más profundo para otro momento.

a) Aspiración final en palabras españolas que terminan en vocal (opcional). Karttunen describe el fenómeno como la introducción de una aspiración final para evitar que las palabras terminen en vocales:

$\begin{array}{ll}\text { bwenob } & \text { "bueno" (SV) } \\ \text { "ì.hoh } & \text { "hijo" (FCP) }\end{array}$

En el maya yucateco hay procesos de debilitamiento de consonantes finales y sustitución de glotales finales ( $c f$. Pfeiler, 1995) y por ello tenemos que tener cuidado al sacar conclusiones sobre el status de la - $b$ final. Además existe mucha variabilidad en la aparencia.

b) Preglotalización de palabras españolas (opcional). Karttunen habla de dos diferentes glotales principales. La primera nunca se pierde, y la otra se considera "débil", porque se pierde en ciertos contextos. Contrario a lo que sostiene Karttunen, hay mucha variabilidad en el uso de la glotal antes de vocal en palabras españolas.

$$
\begin{array}{ll}
\text { "asta / asta } & \text { "hasta" (SV) } \\
\text { espanyol } & \text { "español" (FCP) }
\end{array}
$$

c) Smailus usa la distinción entre $/ r / \mathrm{y} / \bar{r} / \mathrm{del}$ español en las transcripciones. En maya solamente existen algunas palabras con $r$, como turix (libélula). En las palabras españolas la distribución $\operatorname{del} / r /$ y $/ r /$ no queda clara. En Carrillo Puerto se observan más diferencias en la pronunciación de la $r$ :

pero

'ó.mbe "pero" (SV, SA, FCP)

"hombre" (FCP) 


$\begin{array}{ll}\text { crre.rrtik } & \text { "creer" (SV, FCP) } \\ \text { dú.larr } & \text { "dura" (FCP) }\end{array}$

Además, en mi trabajo de campo resultó que los hispanohablantes también pronuncian la $r$ como en inglés $/ R /$, por ejemplo en las palabras tardes o carro.

d) Smailus transcribe $v$ y $b$, pero $\sin$ criterios explícitos, por lo que no se sabe si esta distinción se basa en la diferencia entre la pronunciación de la $b$ glotalizada en el maya y la $b$ del español:

$\begin{array}{ll}\text { favorr } & \text { "favor" (SV) } \\ \text { balor } & \text { "valor" (SV) } \\ \text { vé.tik } & \text { "hacerlo" (FCP) }\end{array}$

e) No se puede ver el acento de las palabras españolas, pero en el caso de anillo queda claro que el acento español en la $i$ se perdió:

$$
\text { á.nyó "anillo" (SV) }
$$

f) En Quintana Roo la pronunciación contiene más sustituciones, como $p$ por $f \circ b$ por $f$ :

$\begin{array}{ll}\text { pá.vorr } & \text { "favor" } \\ \text { plorr } & \text { "flor" } \\ \text { brascitoil } & \text { "frascito-POS" }\end{array}$

Pfeiler (1995) muestra varios procesos de variación fonológica en el maya yucateco hablado en distintas regiones de la península, por ejemplo, la pérdida del cierre glotal final. Sostiene que los procesos son causados o reforzados por el contacto con el español o por el uso de distintos estilos. La motivación para asignar estos efectos al contacto con el español es, según ella, el hecho de que el maya (entre otros) ejerce una influencia en la fonología del español de Yucatán (véanse ejemplos en Pfeiler, 1995). El hecho de que existe influencia del maya se nota, por ejemplo, en la glotalización de consonantes en el es- pañol de la península (Suárez, 1979) y en la pronunciación del español por mayahablantes de la región (Bolle, trabajo de campo, 1996). Eso indica más bien que la fonología del maya es "fuerte" y que sus rasgos no se pierden tan fácilmente. Además, sin hacer un estudio a profundidad de las diferentes regiones e incluso épocas del habla, es díficil saber cuáles son los procesos internos a una lengua y cuáles se refuerzan externamente. Por el hecho de que el maya en sí mismo tiene procesos de contracción y variación, es dificil saber cuál es el factor más importante en los fenómenos mencionados. Lo más probable es que aquí se trate de procesos internos del maya que quizá se fortalecen por la influencia del español.

\section{Conclusión}

Además de una posible influencia fonológica, et uso de los elementos españoles sugiere por lo menos una evidente influencia léxica e incluso morfosintáctica del español. Puede resultar que el maya en algunas instancias en Belice ya presente rasgos de una lengua en proceso de muerte $\mathrm{u}$ obsolescencia lingüística, con mucha restricción de los dominios de habla y con pérdida de elementos morfosintácticos. En este sentido he mencionado la pérdida y variabilidad del uso de los clasificadores numerales p'éel y túul, pero sin un estudio profundo de las lenguas en contacto es dificil confirmar el origen de este proceso.

De hecho, algunas comunidades otrora hablantes de maya ya tienen al español y/o al inglés como lengua primaria (por ejemplo el español en Succotz, Belice). La restricción de dominios lo muestra por ejemplo Pfeiler (1988), entre otros, en cuyo estudio resulta que el maya se usa más en el contexto informal y familiar.

Para hacer un estudio de los cambios en la gramática y de los procesos de convergencia se

Estudios de Cultura Maya. Vol. XXI, 2000 
necesita destacar muy bien el uso de las distintas variantes y estilos. Es necesario establecer, como sugirió Smailus, la pérdida de estructuras del maya mismo. Deberíamos incluso relacionar esto con la evolución interna de la lengua y con el papel que desempeñan los factores externos en el desarrollo del cambio lingüístico, trabajo apenas iniciado en los estudios del maya $y$ al que en el futuro intentaré contribuir sustancialmente (Bolle, en preparación).

\section{Bibliografia}

Barrera Vásquez, Alfredo

1977 "La lengua maya de Yucatán", Enciclopedia yucatanense, vol. 6: Yucatán actual, Edición oficial del gobierno de Yucatán, México, pp. 205-292.

Bolund, O, Nigel.

1992 Colonialismo y resistencia en Belice, ensayos de sociología bistórica, Editorial Grijalbo, México.

BOLLe, JetTE

1994 Sranan Tongo-Nederlands code-wisseling en ontlening [Sranan tongo-holandés cambio de código y préstamos], tesis de maestría, Universidad de Amsterdam.

s.f. "Oral tradition and the effects of language contact" (en preparación).

Briceño Chel, Fidencio

1993 "La cuantificación actual en el maya yucateco", en: Estudios de Lingüística Aplicada, año 12, núms. 19-20: 142-50, unaM, México.

Brockmann, Thomas

1977 a "Ethnic and Racial Relations in Northern Belize", Etbnicity, 4: 246-262.

$1977 \mathrm{~b}$ "Language Communication, and Ethnicity in British Honduras", en William F. Mackey y Jacob Ornstein, Sociolinguistic Studies in BRODY, JILL Language Contact, Mouton, La Haya.

1987 "Particles Borrowed from Spanish as Discourse Markers in Mayan Languages", Anthropological Linguistics, vol. 29, núm. 4: 507-521.
Centro de Investigaciones de Quintana Roo (ciero)

1983 Estudio integral de la frontera México-Belice, 3 tomos, Clero, Chetumal.

FLoRes Farfín, José ANTONIO

1995 Cuatreros somos y toindioma bablamos. Contactos y conflictos entre el nábuatl y el español en el sur de México, tesis de doctorado, Universidad de Amsterdam.

Jiménez Peraza, Pedro César

1982 Lealtad bacia la lengua maya, SEP, INI, México.

KartTunen, Frances

1985 Nabuatl and Maya in Contact with Spanish, Texas Linguistic Forum 26, University of Texas, Austin.

Koenig, Edna LouIsA

1975 Language and Ethnicity in Corozal District, Belize: An Analysis of Code-Switching and Code-Selection, tesis de la Universidad de Texas, Austin (microfilm).

1980 "Ethnicity: the Key Variable in a Case Study of Language Maintenance and Language Shift", Ethnicity, 7: 1-14.

Le PAge, Robert, y André TAbourette-Keller

1985 Acts of Identity, Cambridge University Press, Londres.

Macias Zapata, Gabriel Aaron

1985 "Economía y política entre los mayas icaichés de Quintana Roo, 1893-1980", ponencia presentada en el Tercer Congreso Internacional de Mayistas, 14 de julio de 1995, Chetumal, Quintana Roo, México.

Pfeiler, Barbara

1988 "Yucatán: el uso de dos lenguas en contacto", Estudios de Cultura Maya, xvil: 423-444.

1995 "Variación fonológica en el maya yucateco", en Ramón Arzápalo Marín y Yolanda Lastra (comps.), Vitalidad e influencia de las lenguas indigenas en Latinoamérica, II Coloquio Mauricio Swadesch, unam, México.

1996 El $x e^{\prime} e k^{\prime}$ y la bach maya, cambio y futuro del maya ante la modernidad cultural en Yucatán, UADY, manuscrito.

Smallus, Ortwin

1975 Textos mayas de Belice y Quintana Roo, Indiana suplemento 3, Gebr. Mann Verlag, Berlín.

SuÁrez, Victor M.

1979 El español que se babla en Yucatân, Ediciones de la Universidad de Yucatán, Mérida. 